\title{
Direct brain recordings reveal continuous encoding of structure in random stimuli
}

\author{
Julian Fuhrer ${ }^{\mathrm{a}}$, Kyrre Glette ${ }^{\mathrm{a}}$, Jugoslav Ivanovic ${ }^{\mathrm{b}}$, Pål Gunnar Larsson ${ }^{\mathrm{b}}$, Tristan Bekinschtein ${ }^{\mathrm{c}}$, Silvia Kochen ${ }^{\mathrm{d}}$, \\ Robert T. Knight ${ }^{\mathrm{e}}$, Jim Tørresen ${ }^{\mathrm{a}}$, Anne-Kristin Solbakk ${ }^{\mathrm{b}, \mathrm{f,g}}$, Tor Endestad ${ }^{\mathrm{f}, \mathrm{g}}$, and Alejandro Blenkmann ${ }^{*, \mathrm{~g}}$ \\ ${ }^{a}$ RITMO, Department of Informatics, University of Oslo, 0373 Oslo, Norway \\ ${ }^{\mathrm{b}}$ Department of Neurosurgery, Oslo University Hospital, Rikshospitalet, 0372 Oslo, Norway \\ ${ }^{\mathrm{c}}$ Cambridge Consciousness and Cognition Lab, Department of Psychology, University of Cambridge, Cambridge CB2 3EB, United Kingdom \\ ${ }^{\mathrm{d}}$ Studies in Neurosciences and Complex Systems, National Scientific and Technical Research Council, El Cruce Hospital, Arturo Jauretche \\ National University, Argentina \\ ${ }^{\mathrm{e}}$ Helen Wills Neuroscience Institute and Department of Psychology, University of California, Berkeley, CA 94720, USA \\ ${ }^{\mathrm{f}}$ Department of Neuropsychology, Helgeland Hospital, 8657 Mosjøen, Norway \\ ${ }^{\mathrm{g}}$ RITMO, Department of Psychology, University of Oslo, 0373 Oslo, Norway
}

\begin{abstract}
The brain excels at processing sensory input, even in rich or chaotic environments. Mounting evidence attributes this to the creation of sophisticated internal models of the environment that draw on statistical structures in the unfolding sensory input. Understanding how and where this modeling takes place is a core question in statistical learning. It is unknown how this modeling applies to random sensory signals. Here, we identify conditional relations, through transitional probabilities, as an implicit structure supporting the encoding of a random auditory stream. We evaluate this representation using intracranial electroencephalography recordings by applying information-theoretical principles to high-frequency activity $(75$ to $145 \mathrm{~Hz}$ ). We demonstrate how the brain continuously encodes conditional relations between random stimuli in a network outside of the auditory system following a hierarchical organization including temporal, frontal and hippocampal regions. Our results highlight that hierarchically organized brain areas continuously attempt to order incoming information by maintaining a probabilistic representation of the sensory input, even under random stimuli presentation.
\end{abstract}

Efficient encoding of patterns in ongoing sensory input is critical for survival in an ever-changing environment. The brain is not inherently aware of the underlying structures in the environment and potential regularities in the sensory stream must be assessed with regards to previously encoded regularity (1-3). Regularity encoding involves continuous updating of internal representations of the environment based on statistical structures derived from the sensory signal (4-9). Sensitivity to conditional regularity between events has been observed in humans (10-20) and animals (21-26). Because events in the environment rarely occur independently, this pattern extraction is necessary for fast and efficient processing of sensory information.

A mathematical representation of such conditional regularity is transitional probabilities (TPs). TPs describe how likely one event predicts another, that is, the ratio of the directional co-occurrence of events given their frequency $(6,27-29)$. As an example, experimental studies in infants and adults have shown that the TPs between syllables constitute patterns that facilitate the identification of word-like units (10, 29-33), thus making TP encoding essential for language development $(6,7,28,31,34-36)$.

While the brain's sensitivity to conditional regularities has been observed in experimental studies across sensory domains, the mechanisms underlying this sensitivity remain poorly understood $(6,30,31,37-45)$. Studies on sensory processing and statistical learning have reported engagement of multiple brain structures, suggesting that the perception and learning of statistical regularities is not performed by one neural region, but rather may be supported by multiple regions working in parallel $(31,35,36,43$, for alternative hypotheses 31 ). Sensory modality-general areas, such as the prefrontal cortex and the hippocampus, as well as lower perceptual or modality-specific regions are proposed to subserve this capacity. However,

*E-mail address: a.o.blenkmann@psykologi.uio.no 
detailed knowledge about the brain regions contributing to this dynamic and adaptive process is limited $(6,13,32,36,43,44,46,47)$. In addition to modality specificity, the involved regions depend on the structure of the input (for example, pure tones or linguistic material for auditory stimuli), its complexity (ranging from deterministic to random structures), or whether learning occurs implicitly/automatically or explicitly/attention-dependent $(31,34,35,48)$.

We investigated how different brain regions contribute to statistical learning using the high temporal and spatial resolution of intracranial electroencephalography (iEEG) by examining the information content of high-frequency activity (HFA; 75 to $145 \mathrm{~Hz}$ ) event responses in relationship to TPs between randomly occurring tones. Based on the hypothesis that multiple brain regions engage in auditory statistical learning, we predicted temporal, frontal, insular, and anterior cingulate (ACC) cortices, as well as peri-central sulci and hippocampus to be sensitive to TPs in our implicit learning listening task $(4,31,35$, $36,45)$. We then evaluated whether this sensitivity follows a hierarchical organization within the brain.

\section{Statement of Significance}

Humans are biased to perceive patterns in random sensory signals. However, the underlying neurophysiological mechanisms are unknown. Utilizing the high temporal and spatial precision of intracranial electroencephalography, we found that the brain automatically encodes temporal relationships between events when exposed to random acoustic stimuli. We also revealed a hierarchical structure of brain areas supporting this mechanism. These results suggest that the brain continuously attempts to predict and provide structure from events in the environment, even when they are not behaviorally relevant and have no evident relation between them. Linking the frameworks of statistical learning and predictive coding, our work illuminates an implicit process that might be crucial for the swift detection of patterns and unexpected events in the environment.

\section{Results}

Participants ( $\mathrm{n}=22 ;$ Materials and Methods) listened to a stream of tones where a standard tone alternated with deviant tones $(\mathrm{P}=0.5$; inter-stimulus interval $500 \mathrm{~ms})$. Deviant tones varied relative to the standard in terms of either frequency $(\mathrm{P}=0.1)$, intensity $(\mathrm{P}=0.1)$, perceived sound-source location $(\mathrm{P}=0.1)$, a shortened duration $(\mathrm{P}=0.1)$, or a gap in the middle of the tone $(\mathrm{P}=0.1 ;$ Fig. 1$)$. Within a set of ten tones (five standard tones and five deviant tones), each of the five deviant types was presented once in random order. For deviations in location, intensity, and frequency, two stimuli versions were used $(\mathrm{P}=0.5)$, namely location left/right, intensity low/high, and frequency low/high. This resulted in eight potential deviants. During recording, participants were asked not to pay attention to the sounds while reading a book or magazine. Across the 22 patients, we recorded 1078 channels (mean: 48, range: 12-104). Data were manually cleaned. Noisy or epileptic channels or segments were excluded from the analysis. HFA was reliably extracted from a total of 785 channels within cortical or subcortical structures.

\section{Regions of Interest}

Statements about significance were based on a set of regions of interest (ROIs) that typically engage in auditory processing and statistical learning tasks $(4,31,35,36,45)$, comprising channels in temporal, frontal, hippocampal, insular, pre-central sulci, and ACC cortices, respectively (Fig. 2a, Tab. S1). Average values of the present measure of interest were then determined in the respective analysis for each ROI.

\section{Encoded Information}

We estimated the information content of each deviant tone HFA response in relation to a channel-specific HFA response to standard tones. Hence, the information content in standard responses was used as a reference point to measure the information content in deviant responses. This procedure yielded a normalized measure of encoded information for each deviant response (Fig. 1, bottom; Materials and Methods). Lower values of encoded information suggest that the information content in deviant responses is similar 


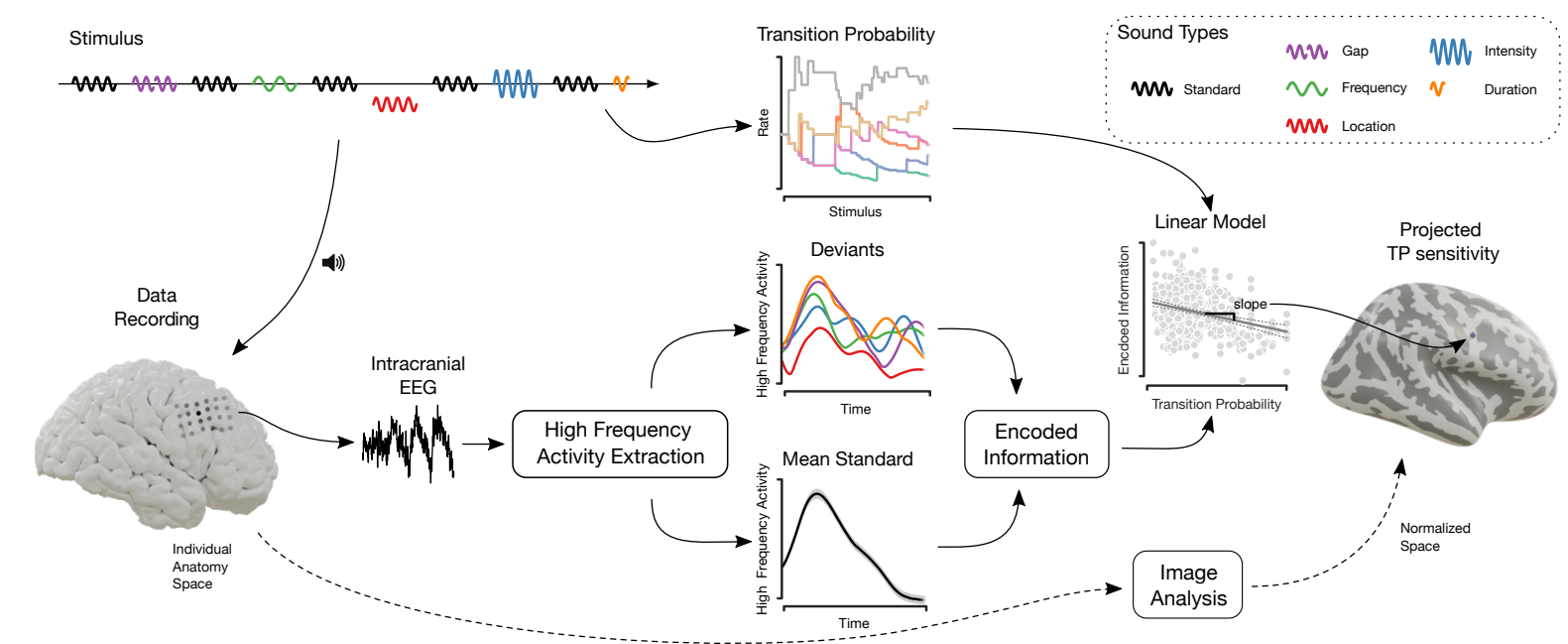

Figure 1: Schematic of the analysis: A multi-feature auditory oddball experiment was presented to participants while recording their eventrelated electrical brain activity through intracranial electrodes. The emerging iEEG signal was then analyzed resulting in HFA responses to standard and deviant tones. Based on the standard responses, a channel-specific mean standard response was computed. Deviant and mean standard responses were then compressed in order to estimate their differences in normalized encoded information. This measure ranged from 0 to 1 with 1 indicating complete similarity between the mean standard and a respective deviant tone. In the next step, a regression analysis between this encoded information measure and the TPs, which were based on the stimulus itself, was performed by means of linear models. After accounting for multiple comparisons, respective channel-specific slope values between encoded information and TP were projected onto the normalized anatomical space to enable comparison across subjects.

to the information content in the standard responses, whereas higher values indicate that a larger amount of information is encoded in the responses to deviants compared to standards.

The spatial distribution of the mean encoded information to deviants per channel is shown in Fig. 2a. The mean encoded information was significantly greater than zero for each ROI (Fig. 2b, one-tailed Wilcoxon signed-rank test, FDR corrected for multiple comparisons). This indicates that, on average, the HFA signals in all the ROIs encoded more information in the deviant than in the standard responses. To further evaluate the level of involvement across ROIs, we computed their median encoded information and performed pairwise Mann-Whitney-Wilcoxon tests (Fig. 2b). Notably, the superior temporal plane and the posterior insula had a significantly greater median value than all other ROIs, suggesting a relatively stronger responsiveness to acoustic deviance.

\section{Transitional Probability Sensitivity}

To determine which brain areas are more sensitive to the temporal relations between stimuli, we evaluated the relationship between encoded information and the TPs of deviant tones through linear models (Fig. 3a, each green dot represents a trial). TPs represent the inter-sound relationships between deviant tones and are determined for each stimulus presentation (that is, they are not uniformly distributed across the stimuli), where higher TP values indicate a higher transition rate (Fig. 1, Material and Methods). The signs of the resulting slope values were inverted and defined as the TP sensitivity. TP sensitivity values acted as an indicator of how sensitive the brain tissue around the channel was towards TPs in the stream of tones. Zero value TP sensitivity of a channel indicates that the encoded information in the deviant responses is not affected by the TPs of the events, whereas higher values imply a higher impact.

In the analysis, 453 out of 785 channels across all subjects showed a significant slope (permutationbased test, FDR corrected for multiple comparisons; Fig. S1). Consequently, these channels tended to increase the amount of encoded information in the HFA response when the likelihood of an event occurrence (i.e., the TP) decreased. The spatial distribution for all TP sensitivity values is illustrated in Fig. 3c. Subsequently, we investigated possible variations in TP sensitivity across ROIs (Fig. 3d). All ROIs' means were significantly greater than zero (one-tailed Wilcoxon signed-rank test; FDR corrected), indicating that, on average, unexpected events (low TP) evoked a higher degree of encoded information. Mann-Whitney-Wilcoxon tests were performed across ROIs to study relative differences in TP sensitivity. Along with the superior temporal plane, channels in the hippocampus showed the greatest median TP sensitivity values. 
(a) ROIs \& Mean Encoded Information
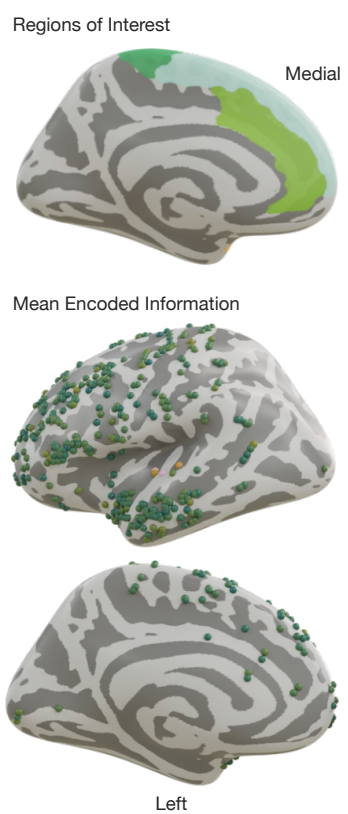
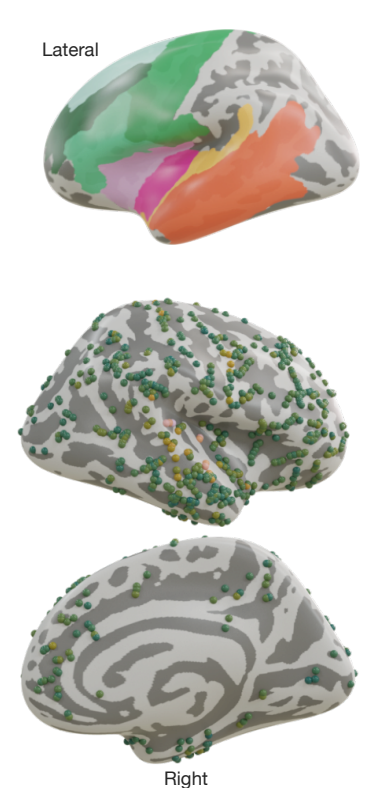

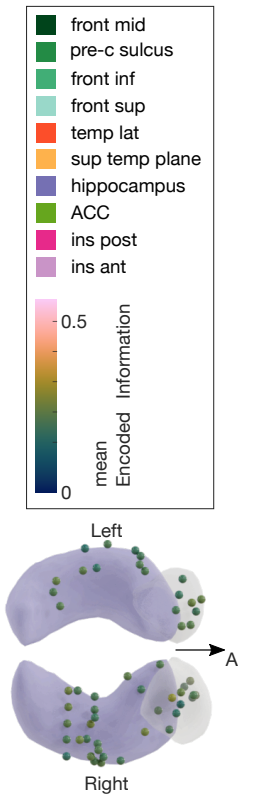

(b) Mean Encoded Information by ROI

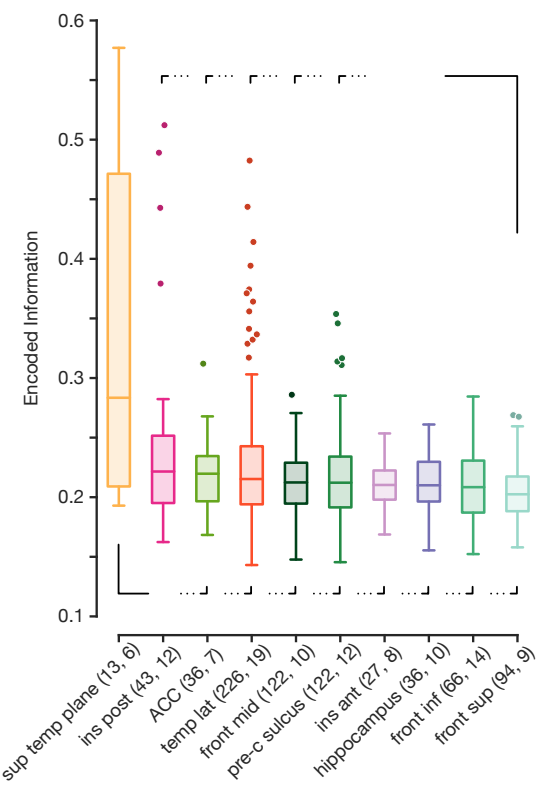

Figure 2: Illustration of the encoded information analysis results. a: Top: ROIs on the inflated brain model (Tab. S1 for full region labels). Bottom: lateral and medial view of the mean encoded information distribution across 22 subjects projected onto the inflated brain model. The image on the bottom right shows the transverse plane of the amygdala (gray) and hippocampus (purple), where "A" stands for the anterior direction. Each sphere represents one channel. b: Box-plots of the ROIs' encoded information. In the axis label, the number of channels (first) and subjects (second) are given for each ROI. The nested brackets indicate a significant difference between median values.

\section{Relation between Mean Encoded Information and Transitional Probability Sensitivity}

We further asked whether brain areas that are more sensitive to deviant stimuli would also be more sensitive to TPs in the stream of tones. Therefore, we examined the relation between slopes (indexing TP sensitivity values) and mean encoded information across all channels. We observed a significant positive correlation between the two regressors (Fig. 3b; linear mixed-effects model with random effects for subjects: $y=\beta_{0}+\beta_{1} x+b_{0}+\epsilon$, with the mean encoded information $y$, the TP sensitivity $x$, the random effect for subjects $b_{0} \sim N\left(0, \sigma_{b}^{2}\right)$ and the observation error $\epsilon \sim N\left(0, \sigma^{2}\right) ; \beta_{0}=0.19,95 \%$ CI $[0.19,0.20]$, $\beta_{1}=0.28,95 \%$ CI $[0.220 .33], p_{\beta_{1}}=0, \sigma_{b}=1.39 \mathrm{e}-2,95 \%$ CI $[9.46 \mathrm{e}-3,2.04 \mathrm{e}-2], \epsilon=4.09 \mathrm{e}-2$, $95 \%$ CI [3.89e-2, 4.29e-2]), reflecting that channels encoding more information are also more sensitive to TPs.

\section{TP sensitivity Along the Hierarchical Pathway}

We utilized inter-areal variations of anatomical hierarchy to investigate changes in TP sensitivity across the brain regions. Anatomical hierarchy can be defined as a global ordering of cortical areas corresponding to characteristic laminar patterns of inter-areal feedforward and feedback projections $(8,50,51)$. Proxied cortical hierarchy levels that quantify these projections across the cortex were obtained from open-access structural magnetic resonance imaging (MRI) datasets from the S1200 subject release (49; Materials and Methods). Methodological constraints in (49) precluded the mapping of the hippocampus in the present analysis. Areas lower in the hierarchy (with predominantly feedforward projections) are primarily associated with primary sensory functions, whereas areas higher in the hierarchy are associated with higher cognitive functions $(8,50,51)$. For each contact point, hierarchy level channel-estimates were determined by taking the average value of all proximal points located within the contact point vicinity. The TP sensitivity significantly correlated with proxied cortical hierarchy levels in the negative direction (Fig. 3f; linear mixed-effects model with random effects for subjects: $y=\beta_{0}+\beta_{1} x+b_{0}+\epsilon$, with proxied hierarchy level $y$, the TP sensitivity $x$, the random effect for subjects $b_{0} \sim N\left(0, \sigma_{b}^{2}\right)$ and the observation error $\epsilon \sim N\left(0, \sigma^{2}\right) ; \beta_{0}=1.25,95 \%$ CI $[1.22,1.28], \beta_{1}=-0.23,95 \%$ CI $[-0.37-0.11], p_{\beta_{1}}=1.11 \mathrm{e}-3$, 
bioRxiv preprint doi: https://doi.org/10.1101/2021.10.01.462295; this version posted October 3, 2021. The copyright holder for this preprint (which was not certified by peer review) is the author/funder. All rights reserved. No reuse allowed without permission.

(a) Example Channels

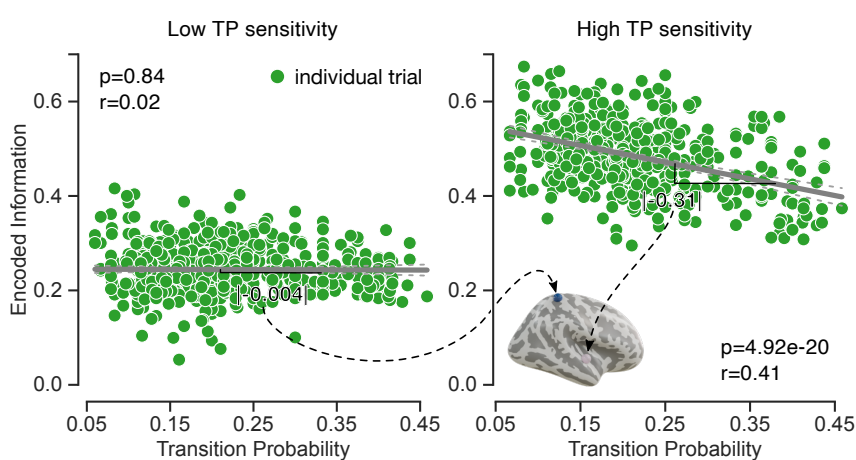

(b) Encoded Information to TP sensitivity

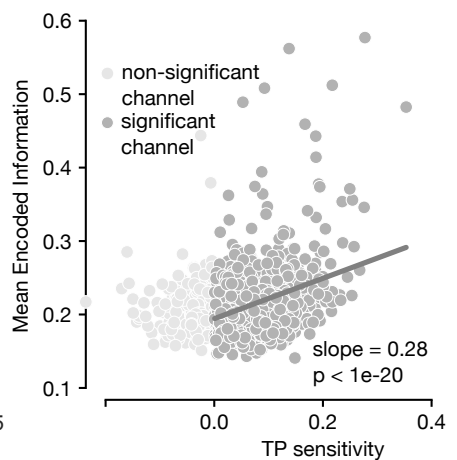

(c) TP sensitivity Across Cortical and Hippocampal Channels
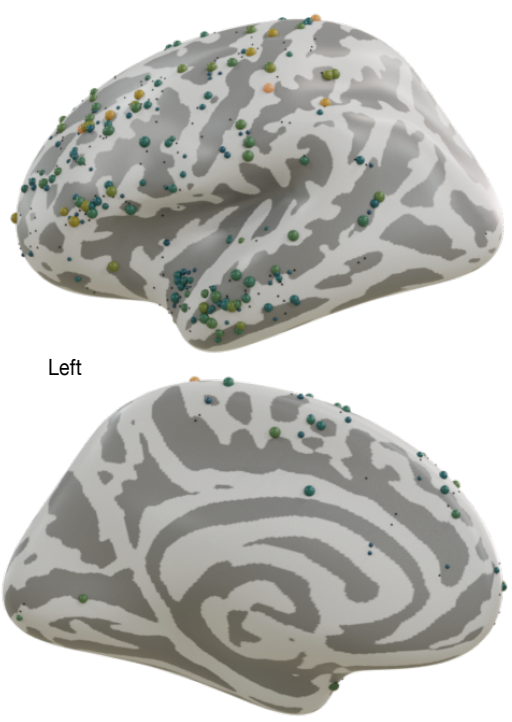

(d) Sensitivity to TP by ROI

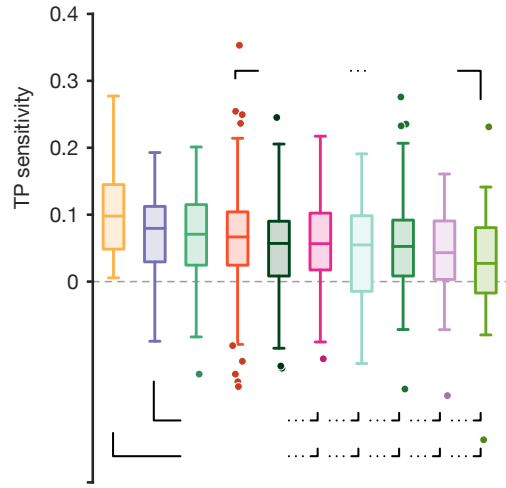

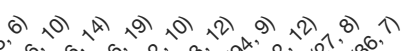

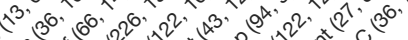

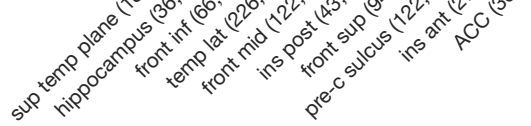

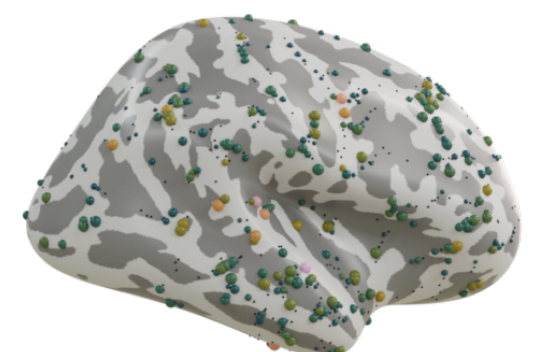

Right
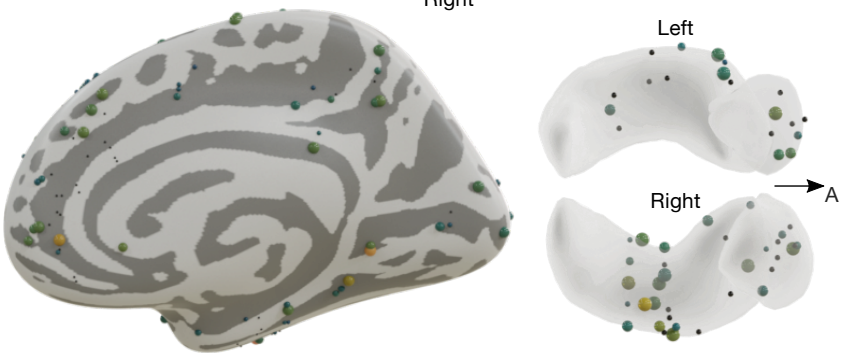

(e) Proxied Hierarchy Levels

(f) Hierarchical Ordering of TP sensitivity
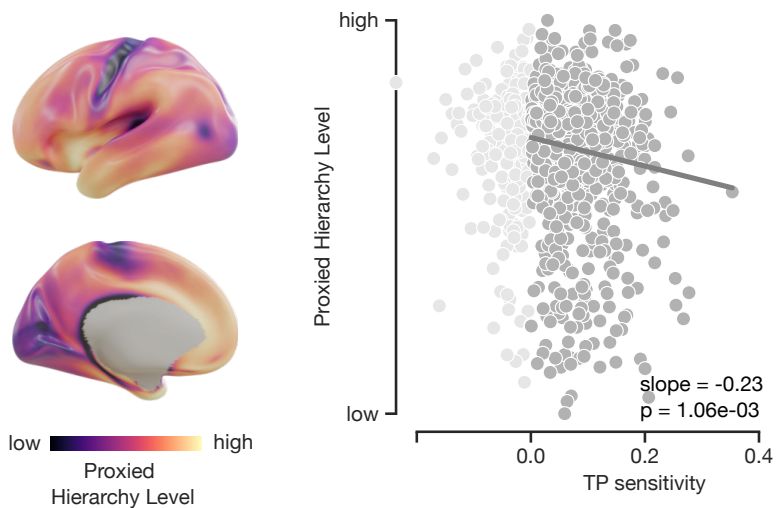

Figure 3: Spatial profile of TP in relation to encoded information. a: Spatial mapping of the slope for two example channels resulting from the robust linear regression between TPs and encoded information (each green dot represents a trial). The slope is an indicator of how sensitive a region underneath a contact point is towards the variation of TP. The second channel shows a negative slope of -0.31 , hence the more often a transition occurs, the more the information encoded in the deviant response decreases. $\mathbf{b}$ : A linear mixed effect model exhibits a positive relationship between the mean encoded information (Fig. 2a) and TP sensitivity. d: TP sensitivity across ROIs. All ROIs show a significant sensitivity to TP. The number of channels and subjects within ROIs are given in parentheses in the axis label. Brackets indicate statistical differences across areas. c: Inflated brain model with lateral and medial views of the right and left hemispheres and a superior view of the amygdala and hippocampus. Each sphere represents a channel projected onto the surface with the colors indicating its TP sensitivity. The size of the spheres indicates the p-value corresponding to the performed regression. The $\mathrm{p}$-values are divided such that each interval contains $1 / 4$ of the p-value set. e: Proxied hierarchy levels obtained from the averaged human T1w/T2w mapping of 1200 subjects (49). f: Proxied hierarchy levels correlated with TP sensitivity through a linear mixed effect model show greater TP sensitivity in areas lower in the hierarchy. 
$\sigma_{b}=5.14 \mathrm{e}-2,95 \%$ CI $[3.58 \mathrm{e}-2,7.37 \mathrm{e}-2], \epsilon=0.10,95 \%$ CI $\left.[0.10,0.11]\right)$. Accordingly, channels with contact points located in areas lower in the hierarchy were more sensitive towards the TP courses.

\section{Discussion}

We studied how humans passively listening to a multi-feature sequence of random sounds implicitly encode conditional relations between the sounds. Crucially, our results show that the auditory system embedded in a distributed hierarchical network, continuously monitors the environment for potential saliency, maintaining and updating a neural representation of temporal relationships between events.

\section{The Brain Encodes TPs in Random Stimulus Sequences}

Participants demonstrated remarkable sensitivity to TPs. From a statistical learning perspective, these findings suggest an implicit learning process in which TPs are internally inferred. On average, more frequent deviant transitions exhibited less encoded information in the HFA responses. Conversely, rarer transitions showed an increase in the encoded information (Fig. 3a \& 3b). These results indicate a steady encoding of TP courses within a randomly ordered sequence of varied auditory stimuli, and are consistent with previous studies using more structured and stationary stimuli $(7,10-20,28)$.

Following the notion of predictive coding, the encoded information in each deviant response can be interpreted as a bottom-up prediction error signal, that is, the amount of information in each novel event that is not explained away by top-down prediction signals $(9,41,52)$. In this sense, low TP events, that is, less expected events, elicited a higher amount of encoded information, and hence larger prediction errors derived from less accurate predictions. Accordingly, this information is used in higher cortical areas to update internal models for future predictions. On the other hand, high TP events, that is, more expected events, elicited a lower amount of encoded information. This generates smaller prediction error signals, and smaller updates of the internal models. Internal representations of TPs between events are fundamental to build useful predictions of upcoming events, rather than simpler frequentist representations (11).

Several studies have shown that the brain implicitly learns patterns within stimulus trains (where patterns were a sub-set of manipulated high TPs among all possible event transitions; $(7,28,45,47))$. Here, we show that the brain is also sensitive to TPs in randomly presented stimuli. The sensitivity to TP within a random sequence indicates that the brain continuously encodes TPs between events in the environment. The likelihood of occurrence of an event given previous knowledge is quantified in the amount of information encoded in the HFA responses of multiple cortical and subcortical areas. This mechanism forms the basis of a statistical learning system wherein the brain integrates each single event into an internal representation of the environment based on the statistical relationship between events. Since a-priori, the presence of patterns within stimuli are unknown, the brain might automatically encode their TP in order to detect potential structure and violations of such. Artificial grammar learning studies, where subjects learn patterns of TP to which they were never exposed before, confirm the relevance of this TP encoding in language learning $(15,31,32,36,53)$.

The encoding of acoustic deviant transitions is anatomically distributed and not exclusively concentrated in auditory cortices (Fig. 3c), which is in accord with previous findings showing that multiple brain regions are involved in statistical learning $(31,35)$. Our results suggest that the automatic process of identifying temporal relationships is subserved by a network consisting of temporal cortices in concert with the hippocampal, inferior frontal, and insular cortices.

Overall, we observed that the higher the sensitivity to TP, the more the encoded information indexed distinct responses between deviants and the channel-specific mean standard (Fig. 3b). Notably, channels in the superior temporal plane showed both the greatest amount of encoded information and the greatest TP sensitivity (Fig. 3d). This suggests a key role of the supratemporal plane in both the deviance detection and the implicit learning of transitions between salient auditory events. This finding is consistent with previous reports about this region being active in conditional statistical learning $(16,36,46,47,54)$. This suggests that classic perceptual areas are not limited to operating on the perceptual processing of individual stimuli, but also contribute to the learning of temporal patterns in streams of stimuli (31). 
Our results indicate that the hippocampus also contributes to encoding of temporal transitions between salient events. However, in contrast to supratemporal responses, hippocampal responses revealed greater sensitivity to TPs than to deviants per se (Fig. 2b). This implies that hippocampal activity may reflect a more generic context sensitivity to the events' probabilistic structures, that is, learning about event occurrences within a given structure itself instead of encoding the probability of their actual occurrence (55). Our results provide new evidence for a role of the hippocampus during implicit learning, consistent with recent suggestions that this area is a rapid supramodal learner of arbitrary or higher-order associations in the sensory environment $(6,15,31,35,36,43-45,47,56,57)$.

We also observed a sensitivity to transitions between salient events in the inferior frontal cortex. Evidence of inferior frontal involvement in statistics-driven learning processes is sparse $(31,36,45)$ and mainly relies on explicit learning studies using fMRI $(1,46)$. However, it is commonly described in the deviance detection literature, where a role of a higher hierarchical node is attributed to this region $(58,59)$.

An unexpected observation was the significant TP sensitivity in the occipital lobe, indicating a contribution to TP encoding of the auditory stimuli. It has been shown that during auditory oddball and statistical learning paradigms, attentional processing can activate visual processing regions, which are typically engaged in the perception of visual objects $(15,60,61)$. When queried, all of our participants reported that they could focus on the reading material and did not pay attention to the tones. Hence, this leaves open whether this auditory occipital activation might also be observable during passive listening tasks and whether this is specific to the sensitivity of our HFA recording. Current evidence is sparse, but two previous on deviance detection during passive listening showed similar occipital effects using fMRI and scalp EEG $(61,62)$.

\section{Hierarchical Organization of TP encoding}

Areas lower in the hierarchy are more sensitive to deviant transitions, and conversely, higher hierarchy locations exhibit lower TP sensitivity (Fig. 3f). Interestingly, our results indicate that the sensitivity to TP was not strictly confined to specific areas, but distributed across multiple brain regions in a hierarchically organized manner.

When taking into consideration the positive correlation between encoded information and TP sensitivity (Fig. 3b), it becomes evident that the lower hierarchical levels, which show a preferential representation of the stimuli, are also more sensitive to changes in TP. Together, these results are in line with studies on the hierarchical visual pathway, which indicate that expectation suppression scaled positively with image preference (63).

In a recent iEEG study presenting 12 syllables within an auditory stream, Henin et al. (15) observed that TPs are encoded in lower-order areas of the superior temporal plane and not in the hippocampus, which uniquely represented the identity (i.e., the specific higher-order chunk such as a word) of their sequences. Therefore, the hippocampus did not appear to engage in forming the neural representation of TPs, but rather performed operations that build upon them. We found the hippocampus to be a main contributor among the cortical areas in detecting TPs. However, our study used passive listening with pure tones, while Henin et al. used active listening with syllables. Even though the hippocampus was not included in the hierarchical organization analysis, the fact that the sensitivity to TP was the second highest predicts a low hierarchical level in relationship to the other brain areas. This result is contradictory with studies that position the hippocampus at the apex of the hierarchical organization, integrating complex information (64). However, the result fits well with previous studies indicating its fundamental role in statistical learning, and encoding stimuli uncertainty $(6,15,31,35,36,43-45,47,55-57,65)$. This suggests that, depending on attentional characteristics and task demands, the hippocampus might operate differently. Due to its domain-general learning mechanisms, possible hippocampal involvement could comprise indirect modulation of lower-level sensory areas or direct computations of hippocampal representations $(31,35)$. 


\section{A Systems Perspective on Deviance Detection}

Our results suggest a main involvement of the superior temporal plane and posterior insula in a deviance detection network (Fig. 2b). Previous studies on auditory deviance detection using iEEG, MEG/EEG source localization, and fMRI have shown similar responses to deviants over the supratemporal plane $(4,37,58,59,62,66-71)$, but detailed information for the insular cortex is sparse. In line with recent reports about its contribution to auditory processing $(67,72)$, we found that the posterior part showed larger differences in encoded information between deviant and standard responses than the anterior part.

We also found that the ACC, middle frontal and pre-central sulcus moderately engaged in change detection. Although not often observed in auditory experiments, activation of these regions has been previously reported in the context of pre-attentive oddball paradigms with frequency (or duration) deviants using EEG $(62,62,69,73)$ or fMRI $(61,71)$. In our study, the ACC appeared to be more active in auditory change detection than TP encoding, generally consistent with previous reports $(62,73)$. It is presumably more involved in cognitive control or error detection, such as recognizing global patterns $(66,68)$. In our pre-attentive paradigm, we speculate that the ACC monitors the high-level structure of individual deviant occurrences rather than the more automatized detection of TPs.

The brain implicitly parses an auditory stimulus with random features. While automatically assessing the deviance of events, the brain simultaneously identifies patterns by encoding conditional relations between events, supporting both statistical learning and predictive coding frameworks. This implicit and hierarchically organized process involves, in addition to pure sensory areas, inferior frontal cortices, hippocampus, and other cortical regions.

\section{Methods}

\section{Stimuli}

A multi-dimensional auditory oddball paradigm was used $(58,59,74,75)$ that included a standard tone and five different deviant tones (Fig. 1). Standards had a duration of $75 \mathrm{~ms}$ with $7 \mathrm{~ms}$ up and down ramps and consisted of three sinusoidal partials of 500,1000 , and $1500 \mathrm{~Hz}$. Deviants varied relative to the standard in the perceived sound-source location (left or right), intensity $( \pm 6 \mathrm{~dB})$, frequency $(550,1100$, and $1650 \mathrm{~Hz}$ or 450,900 , and $1350 \mathrm{~Hz}$ ), gap ( $25 \mathrm{~ms}$ silence in the middle), or by a shortened duration $(1 / 3$ or $25 \mathrm{~ms}$ shorter). Thus there were two stimuli versions for location, intensity, and frequency deviants. During the sequence, each standard tone was followed by a deviant tone. The deviant tone type was set up such that within a set of five consecutive deviants, each of the five types was presented once. In consecutive sets, the same deviant type did not repeat from the end of one set to the beginning of another. For the three deviants that had two stimuli versions, each version occurred equally often $(\mathrm{P}=0.5)$. Except for deviants varying in duration, all tones had a duration of $75 \mathrm{~ms}$ and were presented every $500 \mathrm{~ms}$ in blocks of 5 min consisting of 300 standards and 300 deviants. At the beginning of each block, 15 standards were played. To capture automatic, stimulus-driven processes, participants were asked not to pay attention to the sounds while reading a book or magazine. They completed 3 to 10 blocks, providing at least 1800 trials. Tones were presented through headphones using Psychtoolbox-3 (76).

\section{Participants}

We recorded data from 22 (self-reported) normal-hearing adults with drug-resistant epilepsy who were potential candidates for resective surgery of epileptogenic tissue (mean age 31 years, range 19 to 50 years, 6 female). Patients underwent invasive intracranial electrocorticography (ECoG) or stereoelectroencephalography (SEEG) recordings as part of their pre-surgical evaluation. Intracranial depth electrodes were temporarily implanted to localize the epileptogenic zone and eloquent cortex. The number and placement of electrodes were guided exclusively by clinical requirements. Data were collected at El Cruce Hospital $(\mathrm{n}=15)$ and Oslo University Hospital $(\mathrm{n}=7)$. 


\section{Data Acquisition}

Pre-implantation structural MRI and post-implantation CT scans were acquired for each participant. ECoG or SEEG data were recorded using an Elite (Blackrock NeuroMed LLC, USA), a NicoletOne (Nicolet, Natus Neurology Inc., USA), or an ATLAS (Neuralynx, USA) system with sampling frequencies of 2000, 512, and $16000 \mathrm{~Hz}$, respectively.

\section{Electrode Localization}

Post-implantation CT images were co-registered to pre-implantation MRI images using SPM12 (77). MRI images were processed using the Free-Surfer standard pipeline (78), and individual cortical parcellation images were obtained through the Destrieux atlas (79). Images were spatially normalized to the MNI-152 Template using SPM12 (80), and electrode coordinates were obtained with the iElectrodes Toolbox (81). Anatomical labels were automatically assigned to each contact based on the Destrieux atlas using the aforementioned toolboxes and confirmed by a neurologist/neurosurgeon.

\section{Signal-preprocessing}

Monopolar intracranial EEG recordings were visually inspected and channels or epochs showing epileptiform activity or other abnormal signals were removed. Signals from electrodes located in lesional tissue or tissue that was later resected were also excluded. Bipolar channels were computed as the difference between signals recorded from pairs of neighboring electrodes in the same electrode array. Subsequently, we refer to the bipolar channels as "channels". Data were low-pass filtered at $180 \mathrm{~Hz}$, and line noise was removed using bandstop filters at 50,100, and $150 \mathrm{~Hz}$. Data were then segmented into $2000 \mathrm{~ms}$ epochs ( $750 \mathrm{~ms}$ before and $1250 \mathrm{~ms}$ after tone onset) and demeaned. We visually inspected and rejected epochs after re-referencing. To eliminate any residual artifact, we rejected trials with an amplitude larger than $5 \mathrm{SD}$ from the mean for more than 25 consecutive $\mathrm{Hz}$, or with a power spectral density above $5 \mathrm{SD}$ from the mean for more than 6 consecutive Hz. An average of $35 \%$ of the trials were rejected, resulting in an average of 1592 trials analyzed per patient (range 728 to 3723). Data were resampled to $1000 \mathrm{~Hz}$. Preprocessing and statistical analysis were performed in Matlab using the Fieldtrip Toolbox (82) and custom code. To obtain the HFA, preprocessed data were bandpass filtered into eight bands of $10 \mathrm{~Hz}$ bandwidth ranging from 75 to $145 \mathrm{~Hz}$. The Hilbert transform was then applied to each filtered signal to obtain the complex-valued analytic time series. For more information, see (67, Chap. 2).

\section{Information Distance}

The notion of applying algorithmic complexity to EEG signals has been successfully employed to discriminate between states of consciousness (83-86). One such algorithmic complexity is the Kolmogorov Complexity (K-complexity), which can be described as the ultimate compressed version or minimum description length of an object, delivering its absolute information content (87). If the minimum description length is short (long), the object can be described as "simple" ("complex"). The theoretically ideal $\mathrm{K}$-complexity value of an object cannot be computed and is often heuristically estimated, obtaining an upper-bound approximation. One possible estimate constitutes conventional lossless data compression programs such as for example gzip and bzip2 $(87,88)$. To compare different complexity estimates, it is crucial to apply the same procedure for each object.

Based on the K-complexity, various metrics have been derived. One instance is the Normalized Information Distance (NID) or its estimation counterpart, the Normalized Compression Distance (NCD). The NCD allows the comparison of different pairs of objects with each other and suggests similarity based on their dominating features (or a mixture of sub-features) $(89,90)$. For a pair of strings $(x, y)$, the $\operatorname{NCD}(x, y)$ can be defined as

$$
\operatorname{NCD}(x, y)=\frac{C(x y)-\min (C(x), C(y))}{\max (C(x), C(y))},
$$

with $C(x y)$ denoting the compressed size of the concatenation of $x$ and $y, C(x)$ standing for the compressed size of $x$, and $C(y)$ for the compressed size of $y$ (87-90). Further, the NCD is non-negative, that 
is, it is $0 \leq \mathrm{NCD}(x, y) \leq 1+\epsilon$, where the $\epsilon$ accounts for the imperfection of the employed compression technique. Small NCD values suggest similar objects, and high values suggest rather different objects.

We used the NCD metric to compare the eight deviant types' responses to the mean standard response for each channel (Fig. 1). For the compressor program, we used gzip or bzip2, both showing similar results. Before the compression of an HFA response, the response itself was discretized into a grid of 128 bins. The indices vector of the bins containing the respective signal values was then compressed with a compression routine based on Python's standard library.

\section{Transitional Probability}

We estimated conditional statistics describing the inter-sound relationship through transitional probabilities between deviant tones. A TP matrix for each time step was estimated by cumulating all possible adjacent deviant transitions, yielding a stochastic matrix of size $\mathbb{R}^{8 \times 8}(13,28,29)$.

\section{Anatomical Hierarchy}

Human T1w/T2w maps were obtained from the Human Connectome Project (HCP) (49). The maps were then converted from the surface-based CIFTI file format to the MNI-152 inflated cortical surface template with Workbench Command (91). The structural neuroimaging maps are suggested to be a measure sensitive to regional variation in cortical gray-matter myelin content (50). One function of myelin might be to act as an inhibitor of intra-cortical circuit plasticity. Early sensory areas may require less plasticity, hence more myelination, and hierarchically higher association areas, in turn, have less myelination, presumably enabling greater plasticity (92). Accordingly, T1w/T2w maps may serve as a non-invasive proxy of anatomical hierarchy across the human cortex through an inverse relationship. The anatomical hierarchy can be defined as a global ordering of cortical areas corresponding to characteristic laminar patterns of inter-areal projections $(8,50,51)$. In order to directly work with the hierarchy ordering, T1w/T2w maps were inverted and normalized to the value range of our data set.

\section{Statistical Analysis}

For the statistical analysis, the first 30 trials of each recording block were disregarded. To estimate the TP sensitivity, the distinct tone types were grouped together into one variable. Subsequently, robust linear regression was performed in Matlab (Fig. 1\&3a). TP values greater than 0.7 were excluded. For the regression itself, an alpha value of 0.05 was considered significant. To correct for multiple comparisons, false discovery rate (FDR) adjustment was applied with an FDR of 0.05. Further, because our linear regression model examined the relationship between information content and TPs of all adjacent deviant transitions we performed surrogate data testing with shuffled surrogates for uncorrelated noise (93).

\section{Ethics approval and consent to participate}

This study was approved by the Research Ethics Committee of El Cruce Hospital, Argentina, and the Regional Committees for Medical and Health Research Ethics, Region North Norway. Patients gave written informed consent prior to participation.

\section{Availability of data and materials}

The patients' datasets analyzed for the current study are not publicly available due to our ethical approval conditions that do not permit public archiving of anonymized study data. Materials for the experimental scripts and stimuli, and custom analysis code is available at osf.io/2n6c9.

\section{Acknowledgments}

We thank the patients for kindly participating in our study. We want to express our gratitude to the EEG technicians at El Cruce Hospital and Oslo University Hospital-Rikshospitalet for their support. We thank Yamil Vidal, Fernando Rosas, and RITMO colleagues for rich discussions. This work was 
bioRxiv preprint doi: https://doi.org/10.1101/2021.10.01.462295; this version posted October 3, 2021. The copyright holder for this preprint (which was not certified by peer review) is the author/funder. All rights reserved. No reuse allowed without permission.

partly supported by the Research Council of Norway (RCN) through its Centres of Excellence scheme project number 262762, RCN project number 240389, NINDS Grant R37NS21135, NIMH CONTE Center P50MH109429, and Brain Initiative U01-NS108916.

\section{References}

[1] S. A. Huettel, P. B. Mack, and G. McCarthy, "Perceiving patterns in random series: dynamic processing of sequence in prefrontal cortex," Nature Neuroscience, vol. 5, no. 5, pp. 1546-1726, 2002.

[2] R. Falk and C. Konold, "Making Sense of Randomness: Implicit Encoding as a Basis for Judgment," Psychological Review, vol. 104, no. 2, pp. 301-318, 1997.

[3] U. Hahn and P. A. Warren, "Perceptions of Randomness: Why Three Heads Are Better Than Four," Psychological Review, vol. 116, no. 2, pp. 454-461, 2009.

[4] S. Dürschmid, E. Edwards, C. Reichert, C. Dewar, H. Hinrichs, H.-J. Heinze, H. E. Kirsch, S. S. Dalal, L. Y. Deouell, and R. T. Knight, "Hierarchy of prediction errors for auditory events in human temporal and frontal cortex," Proceedings of the National Academy of Sciences, vol. 113, no. 24, pp. 6755-6760, 2016.

[5] P. Paavilainen, "The mismatch-negativity ( $\mathrm{mmn}$ ) component of the auditory event-related potential to violations of abstract regularities: A review," International Journal of Psychophysiology, vol. 88, no. 2, pp. 109 - 123, 2013.

[6] E. D. Thiessen, "What's statistical about learning? insights from modelling statistical learning as a set of memory processes," Philosophical Transactions of the Royal Society B: Biological Sciences, vol. 372, no. 1711, p. 20160056, 2017.

[7] J. R. Saffran, R. N. Aslin, and E. L. Newport, "Statistical learning by 8-month-old infants," Science, vol. 274, no. 5294, pp. 1926-1928, 1996.

[8] A. Bastos, J. Vezoli, C. Bosman, J.-M. Schoffelen, R. Oostenveld, J. Dowdall, P. De Weerd, H. Kennedy, and P. Fries, "Visual areas exert feedforward and feedback influences through distinct frequency channels," Neuron, vol. 85, no. 2, pp. $390-401,2015$.

[9] K. Friston, “The free-energy principle: A unified brain theory?” Nature Reviews Neuroscience, vol. 11, no. 2, pp. 127-138, 2010.

[10] S. Koelsch, T. Busch, S. Jentschke, and M. Rohrmeier, "Under the hood of statistical learning: A statistical mmn reflects the magnitude of transitional probabilities in auditory sequences," Scientific Reports, vol. 6, pp. 2045-2322, 022016.

[11] M. Mittag, R. Takegata, and I. Winkler, "Transitional probabilities are prioritized over stimulus/pattern probabilities in auditory deviance detection: Memory basis for predictive sound processing," Journal of Neuroscience, vol. 36, no. 37, pp. 9572-9579, 2016.

[12] H. Higashi, T. Minami, and S. Nakauchi, "Variation in event-related potentials by state transitions," Frontiers in Human Neuroscience, vol. 11, p. 75, 2017. [Online]. Available: https://www.frontiersin.org/article/10.3389/fnhum.2017.00075

[13] F. Meyniel, M. Maheu, and S. Dehaene, "Human inferences about sequences: A minimal transition probability model," PLOS Computational Biology, vol. 12, no. 12, pp. 1-26, 2016.

[14] M. K. Leonard, K. E. Bouchard, C. Tang, and E. F. Chang, "Dynamic encoding of speech sequence probability in human temporal cortex," Journal of Neuroscience, vol. 35, no. 18, pp. 7203-7214, 2015.

[15] S. Henin, N. B. Turk-Browne, D. Friedman, A. Liu, P. Dugan, A. Flinker, W. Doyle, O. Devinsky, and L. Melloni, "Learning hierarchical sequence representations across human cortex and hippocampus," Science Advances, vol. 7, no. 8, 2021.

[16] F. Meyniel and S. Dehaene, "Brain networks for confidence weighting and hierarchical inference during probabilistic learning," Proceedings of the National Academy of Sciences, vol. 114, no. 19, pp. E3859-E3868, 2017.

[17] P. Domenech and J.-C. Dreher, "Decision threshold modulation in the human brain," Journal of Neuroscience, vol. 30 , no. 43, pp. 14305-14317, 2010.

[18] P. Maguire, P. Moser, R. Maguire, and M. T. Keane, "Seeing patterns in randomness: A computational model of surprise," Topics in Cognitive Science, vol. 11, no. 1, pp. 103-118, 2019.

[19] M. Maheu, F. Meyniel, and S. Dehaene, "Rational arbitration between statistics and rules in human sequence learning," bioRxiv, vol. 1, no. 1, 2020

[20] F. Meyniel, D. Schlunegger, and S. Dehaene, "The sense of confidence during probabilistic learning: A normative account," PLOS Computational Biology, vol. 11, no. 6, pp. 1-25, 062015.

[21] A. Yaron, I. Hershenhoren, and I. Nelken, "Sensitivity to complex statistical regularities in rat auditory cortex," Neuron, vol. 76, no. 3, pp. $603-615,2012$.

[22] D. Lipkind, G. Marcus, D. Bemis, K. Sasahara, N. Jacoby, M. Takahasi, K. Suzuki, O. Fehér, P. Ravbar, K. Okanoya, and O. Tchernichovski, "Stepwise acquisition of vocal combinatorial capacity in songbirds and human infants," Nature, vol. 498, pp. 104-108, 052013.

[23] M. Takahasi, H. Yamada, and K. Okanoya, "Statistical and prosodic cues for song segmentation learning by bengalese finches (lonchura striata var. domestica)," Ethology, vol. 116, no. 6, pp. 481-489, 2010.

[24] J. E. Markowitz, E. Ivie, L. Kligler, and T. J. Gardner, "Long-range order in canary song," PLOS Computational Biology, vol. 9, no. 5, pp. 1-12, 052013.

[25] J. Chen and C. ten Cate, "Zebra finches can use positional and transitional cues to distinguish vocal element strings," Behavioural Processes, vol. 117, pp. 29 - 34, 2015, cause and Function in Behavioral Biology: A tribute to Jerry Hogan.

[26] K. Lu and D. S. Vicario, "Statistical learning of recurring sound patterns encodes auditory objects in songbird forebrain," Proceedings of the National Academy of Sciences, vol. 111, no. 40, pp. 14 553-14 558, 2014. 
bioRxiv preprint doi: https://doi.org/10.1101/2021.10.01.462295; this version posted October 3, 2021. The copyright holder for this preprint (which was not certified by peer review) is the author/funder. All rights reserved. No reuse allowed without permission.

[27] M. Maheu, S. Dehaene, and F. Meyniel, "Brain signatures of a multiscale process of sequence learning in humans," eLife, vol. 8, p. e41541, 2019.

[28] B. Pelucchi, J. F. Hay, and J. R. Saffran, "Learning in reverse: Eight-month-old infants track backward transitional probabilities," Cognition, vol. 113, no. 2, pp. $244-247,2009$.

[29] S. P. Thompson and E. L. Newport, "Statistical learning of syntax: The role of transitional probability," Language Learning and Development, vol. 3, no. 1, pp. 1-42, 2007.

[30] J. R. Saffran, "Statistical language learning in infancy," Child Development Perspectives, vol. 14, no. 1, pp. 49-54, 2020.

[31] C. M. Conway, "How does the brain learn environmental structure? ten core principles for understanding the neurocognitive mechanisms of statistical learning," Neuroscience \& Biobehavioral Reviews, vol. 112, pp. 279 - 299, 2020.

[32] S. Dehaene, F. Meyniel, C. Wacongne, L. Wang, and C. Pallier, "The neural representation of sequences: From transition probabilities to algebraic patterns and linguistic trees," Neuron, vol. 88, no. 1, pp. 2 - 19, 2015.

[33] L. H. Arnal and A.-L. Giraud, "Cortical oscillations and sensory predictions," Trends in Cognitive Sciences, vol. 16, no. 7, pp. 390-398, 2012.

[34] J. R. Saffran and N. Z. Kirkham, "Infant statistical learning," Annual Review of Psychology, vol. 69, no. 1, pp. 181-203, 2018.

[35] R. Frost, B. C. Armstrong, N. Siegelman, and M. H. Christiansen, "Domain generality versus modality specificity: The paradox of statistical learning," Trends in Cognitive Sciences, vol. 19, no. 3, pp. 117-125, 2015.

[36] J. N. Williams, "The neuroscience of implicit learning," Language Learning, vol. 70, no. S2, pp. 255-307, 2020.

[37] M. Heilbron and M. Chait, "Great expectations: Is there evidence for predictive coding in auditory cortex?" Neuroscience, vol. 389, pp. 54-73, 2018, sensory Sequence Processing in the Brain.

[38] S. L. Denham and I. Winkler, "Predictive coding in auditory perception: challenges and unresolved questions," European Journal of Neuroscience, vol. 51, no. 5, pp. 1151-1160, 2020.

[39] G. V. Carbajal and M. S. Malmierca, "The neuronal basis of predictive coding along the auditory pathway: From the subcortical roots to cortical deviance detection," Trends in Hearing, vol. 22, p. 2331216518784822, 2018.

[40] J. Rubin, N. Ulanovsky, I. Nelken, and N. Tishby, "The representation of prediction error in auditory cortex," PLOS Computational Biology, vol. 12, no. 8, pp. 1-28, 082016.

[41] A. Clark, "Whatever next? predictive brains, situated agents, and the future of cognitive science," Behavioral and Brain Sciences, vol. 36, no. 3, p. 181-204, 2013.

[42] J. Daltrozzo and C. M. Conway, "Neurocognitive mechanisms of statistical-sequential learning: what do event-related potentials tell us?" Frontiers in Human Neuroscience, vol. 8, p. 437, 2014.

[43] N. V. Covington, S. Brown-Schmidt, and M. C. Duff, "The necessity of the hippocampus for statistical learning," Journal of Cognitive Neuroscience, vol. 30, no. 5, pp. 680-697, 2018.

[44] A. C. Schapiro, E. Gregory, B. Landau, M. McCloskey, and N. B. Turk-Browne, "The Necessity of the Medial Temporal Lobe for Statistical Learning," Journal of Cognitive Neuroscience, vol. 26, no. 8, pp. 1736-1747, aug 2014.

[45] N. Barascud, M. T. Pearce, T. D. Griffiths, K. J. Friston, and M. Chait, "Brain responses in humans reveal ideal observerlike sensitivity to complex acoustic patterns," Proceedings of the National Academy of Sciences, vol. 113, no. 5, pp. E616-E625, 2016.

[46] E. A. Karuza, E. L. Newport, R. N. Aslin, S. J. Starling, M. E. Tivarus, and D. Bavelier, "The neural correlates of statistical learning in a word segmentation task: An fMRI study," Brain and Language, vol. 127, no. 1, pp. 46-54, 2013.

[47] T. Daikoku, "Neurophysiological markers of statistical learning in music and language: Hierarchy, entropy, and uncertainty," Brain Sciences, vol. 8, p. 114, 062018.

[48] R. Frost, B. C. Armstrong, and M. H. Christiansen, "Statistical learning research: A critical review and possible new directions," Psychological Bulletin, vol. 145, no. 12, pp. 1128-1153, 2019.

[49] M. Glasser, T. Coalson, E. Robinson, C. Hacker, J. Harwell, E. Yacoub, K. Ugurbil, J. Andersson, C. Beckmann, M. Jenkinson, S. Smith, and D. Van Essen, "A multi-modal parcellation of human cerebral cortex," Nature, vol. 536, pp. 171-178, 072016.

[50] J. B. Burt, M. Demirtaş, W. J. Eckner, N. M. Navejar, J. L. Ji, W. J. Martin, A. Bernacchia, A. Anticevic, and J. D. Murray, "Hierarchy of transcriptomic specialization across human cortex captured by structural neuroimaging topography," Nature neuroscience, vol. 21, no. 9, p. 1251-1259, September 2018.

[51] N. T. Markov, J. Vezoli, P. Chameau, A. Falchier, R. Quilodran, C. Huissoud, C. Lamy, P. Misery, P. Giroud, S. Ullman, P. Barone, C. Dehay, K. Knoblauch, and H. Kennedy, "Anatomy of hierarchy: Feedforward and feedback pathways in macaque visual cortex," Journal of Comparative Neurology, vol. 522, no. 1, pp. 225-259, 2014.

[52] K. S. Walsh, D. P. McGovern, A. Clark, and R. G. O'Connell, "Evaluating the neurophysiological evidence for predictive processing as a model of perception," Annals of the New York Academy of Sciences, vol. 1464, no. 1, pp. 242-268, 2020.

[53] Y. Vidal, E. Viviani, D. Zoccolan, and D. Crepaldi, "A general-purpose mechanism of visual feature association in visual word identification and beyond," Current Biology, vol. 31, no. 6, pp. 1261-1267.e3, 2021.

[54] K. McNealy, J. C. Mazziotta, and M. Dapretto, "Cracking the language code: Neural mechanisms underlying speech parsing," Journal of Neuroscience, vol. 26, no. 29, pp. 7629-7639, 2006.

[55] B. A. Strange, A. Duggins, W. Penny, R. J. Dolan, and K. J. Friston, "Information theory, novelty and hippocampal responses: Unpredicted or unpredictable?” Neural Networks, vol. 18, no. 3, pp. 225-230, 2005.

[56] N. B. Turk-Browne, B. J. Scholl, M. M. Chun, and M. K. Johnson, "Neural evidence of statistical learning: Efficient detection of visual regularities without awareness," Journal of Cognitive Neuroscience, vol. 21, no. 10, pp. 1934-1945, 2009.

[57] A. C. Schapiro, N. B. Turk-Browne, K. A. Norman, and M. M. Botvinick, "Statistical learning of temporal community structure in the hippocampus," Hippocampus, vol. 26, no. 1, pp. 3-8, 2016. 
bioRxiv preprint doi: https://doi.org/10.1101/2021.10.01.462295; this version posted October 3, 2021. The copyright holder for this preprint (which was not certified by peer review) is the author/funder. All rights reserved. No reuse allowed without permission.

[58] H. N. Phillips, A. Blenkmann, L. E. Hughes, T. A. Bekinschtein, and J. B. Rowe, "Hierarchical organization of frontotemporal networks for the prediction of stimuli across multiple dimensions," Journal of Neuroscience, vol. 35, no. 25, pp. 9255-9264, 2015.

[59] H. N. Phillips, A. Blenkmann, L. E. Hughes, S. Kochen, T. A. Bekinschtein, Cam-CAN, and J. B. Rowe, "Convergent evidence for hierarchical prediction networks from human electrocorticography and magnetoencephalography," Cortex, vol. 82, pp. 192-205, 2016.

[60] J. J. McDonald, V. S. Störmer, A. Martinez, W. Feng, and S. A. Hillyard, "Salient sounds activate human visual cortex automatically," Journal of Neuroscience, vol. 33, no. 21, pp. 9194-9201, 2013.

[61] C. Justen and C. Herbert, "The spatio-temporal dynamics of deviance and target detection in the passive and active auditory oddball paradigm: A sLORETA study," BMC Neuroscience, vol. 19, no. 1, pp. 1-18, 2018.

[62] S. Molholm, A. Martinez, W. Ritter, D. C. Javitt, and J. J. Foxe, "The neural circuitry of pre-attentive auditory changedetection: An fMRI study of pitch and duration mismatch negativity generators," Cerebral Cortex, vol. 15, no. 5, pp. $545-551,2005$

[63] D. Richter, M. Ekman, and F. P. de Lange, "Suppressed sensory response to predictable object stimuli throughout the ventral visual stream," Journal of Neuroscience, vol. 38, no. 34, pp. 7452-7461, 2018.

[64] P. Lavenex and D. G. Amaral, "Hippocampal-neocortical interaction: A hierarchy of associativity," Hippocampus, vol. 10 , no. 4, pp. 420-430, 2000.

[65] L. Harrison, A. Duggins, and K. Friston, "Encoding uncertainty in the hippocampus," Neural Networks, vol. 19, no. 5, pp. 535-546, 2006

[66] T. A. Bekinschtein, S. Dehaene, B. Rohaut, F. Tadel, L. Cohen, and L. Naccache, "Neural signature of the conscious processing of auditory regularities," Proceedings of the National Academy of Sciences, vol. 106, no. 5, pp. 1672-1677, 2009.

[67] A. O. Blenkmann, S. Collavini, J. Lubell, A. Llorens, I. Funderud, J. Ivanovic, P. G. Larsson, T. R. Meling, T. Bekinschtein, S. Kochen, T. Endestad, R. T. Knight, and A.-K. Solbakk, "Auditory deviance detection in the human insula: An intracranial eeg study," Cortex, vol. 121, pp. 189 - 200, 2019.

[68] C. Wacongne, E. Labyt, V. van Wassenhove, T. Bekinschtein, L. Naccache, and S. Dehaene, "Evidence for a hierarchy of predictions and prediction errors in human cortex," Proceedings of the National Academy of Sciences, vol. 108, no. 51, pp. 20754-20 759, 2011.

[69] C. F. Doeller, B. Opitz, A. Mecklinger, C. Krick, W. Reith, and E. Schröger, "Prefrontal cortex involvement in preattentive auditory deviance detection:: neuroimaging and electrophysiological evidence," NeuroImage, vol. 20, no. 2, pp. 1270 1282,2003

[70] M. H. Giard, J. Lavikahen, K. Reinikainen, F. Perrin, O. Bertrand, J. Pernier, and R. Näätänen, "Separate representation of stimulus frequency, intensity, and duration in auditory sensory memory: An event-related potential and dipole-model analysis," Journal of Cognitive Neuroscience, vol. 7, no. 2, pp. 133-143, 1995.

[71] L. Y. Deouell, "The frontal generator of the mismatch negativity revisited," Journal of Psychophysiology, vol. 21, no. 3-4, pp. 188-203, 2007.

[72] Y. Zhang, W. Zhou, S. Wang, Q. Zhou, H. Wang, B. Zhang, J. Huang, B. Hong, and X. Wang, "The Roles of Subdivisions of Human Insula in Emotion Perception and Auditory Processing," Cerebral Cortex, vol. 29, no. 2, pp. 517-528, 012018.

[73] C. Hofmann-Shen, B. O. Vogel, M. Kaffes, A. Rudolph, E. C. Brown, C. Tas, M. Brüne, and A. H. Neuhaus, "Mapping adaptation, deviance detection, and prediction error in auditory processing," NeuroImage, vol. 207, p. 116432, 2020.

[74] R. Näätänen, S. Pakarinen, T. Rinne, and R. Takegata, "The mismatch negativity ( $\mathrm{mmn}$ ): towards the optimal paradigm," Clinical Neurophysiology, vol. 115, no. 1, pp. 140-144, 2004.

[75] L. E. Hughes and J. B. Rowe, "The impact of neurodegeneration on network connectivity: A study of change detection in frontotemporal dementia," Journal of Cognitive Neuroscience, vol. 25, no. 5, pp. 802-813, 2013.

[76] M. Kleiner, D. Brainard, D. Pelli, A. Ingling, R. Murray, and C. Broussard, "What's new in psychtoolbox-3," Perception, vol. 36 , no. 14 , pp. 1-16, 2007.

[77] C. Studholme, D. Hill, and D. Hawkes, "An overlap invariant entropy measure of 3d medical image alignment," Pattern Recognition, vol. 32, no. 1, pp. $71-86,1999$.

[78] A. M. Dale, B. Fischl, and M. I. Sereno, "Cortical surface-based analysis: I. segmentation and surface reconstruction," Neurolmage, vol. 9, no. 2, pp. 179 - 194, 1999.

[79] C. Destrieux, B. Fischl, A. Dale, and E. Halgren, "Automatic parcellation of human cortical gyri and sulci using standard anatomical nomenclature," NeuroImage, vol. 53, no. 1, pp. 1 - 15, 2010.

[80] J. Ashburner and K. Friston, "Unified segmentation," NeuroImage, vol. 26, no. 3, pp. 839 - 851, 2005.

[81] A. O. Blenkmann, H. N. Phillips, J. P. Princich, J. B. Rowe, T. A. Bekinschtein, C. H. Muravchik, and S. Kochen, "ielectrodes: A comprehensive open-source toolbox for depth and subdural grid electrode localization," Frontiers in Neuroinformatics, vol. 11, p. 14, 2017.

[82] R. Oostenveld, P. Fries, E. Maris, and J.-M. Schoffelen, "Fieldtrip: Open source software for advanced analysis of meg, eeg, and invasive electrophysiological data," Computational intelligence and neuroscience, vol. 2011, p. 156869, 012011.

[83] J. D. Sitt, J.-R. King, I. El Karoui, B. Rohaut, F. Faugeras, A. Gramfort, L. Cohen, M. Sigman, S. Dehaene, and L. Naccache, "Large scale screening of neural signatures of consciousness in patients in a vegetative or minimally conscious state," Brain, vol. 137, no. 8, pp. 2258-2270, 062014.

[84] A. Canales-Johnson, A. J. Billig, F. Olivares, A. Gonzalez, M. d. C. Garcia, W. Silva, E. Vaucheret, C. Ciraolo, E. Mikulan, A. Ibanez, D. Huepe, V. Noreika, S. Chennu, and T. A. Bekinschtein, "Dissociable Neural Information Dynamics of Perceptual Integration and Differentiation during Bistable Perception," Cerebral Cortex, vol. 30, no. 8, pp. 4563-4580, 032020. 
[85] M. Schartner, A. Pigorini, S. A. Gibbs, G. Arnulfo, S. Sarasso, L. Barnett, L. Nobili, M. Massimini, A. K. Seth, and A. B. Barrett, "Global and local complexity of intracranial EEG decreases during NREM sleep," Neuroscience of Consciousness, vol. 2017, no. 1, 012017.

[86] M. Schartner, A. Seth, Q. Noirhomme, M. Boly, M.-A. Bruno, S. Laureys, and A. Barrett, "Complexity of multidimensional spontaneous eeg decreases during propofol induced general anaesthesia," PLOS ONE, vol. 10, no. 8, pp. $1-21,082015$.

[87] M. Li and P. Vitányi, An Introduction to Kolmogorov Complexity and Its Applications, 3rd ed., ser. Texts in Computer Science. Springer New York, 2008.

[88] P. M. B. Vitányi, F. J. Balbach, R. L. Cilibrasi, and M. Li, Normalized Information Distance. Boston, MA: Springer, 2009 , pp. 45-82.

[89] M. Li, X. Chen, X. Li, B. Ma, and P. Vitanyi, “The similarity metric,” IEEE Transactions on Information Theory, vol. 50, no. 12 , pp. 3250-3264, 2004.

[90] R. L. Clibrasi and P. M. B. Vitányi, "Clustering by compression," IEEE Transactions on Information Theory, vol. 51, no. 4, pp. 1523-1545, 2005.

[91] D. Marcus, J. Harwell, T. Olsen, M. Hodge, M. Glasser, F. Prior, M. Jenkinson, T. Laumann, S. Curtiss, and D. Van Essen, "Informatics and data mining tools and strategies for the human connectome project," Frontiers in Neuroinformatics, vol. 5, p. 4, 2011.

[92] M. F. Glasser, M. S. Goyal, T. M. Preuss, M. E. Raichle, and D. C. Van Essen, "Trends and properties of human cerebral cortex: Correlations with cortical myelin content," NeuroImage, vol. 93, pp. 165 - 175, 2014.

[93] G. Lancaster, D. Iatsenko, A. Pidde, V. Ticcinelli, and A. Stefanovska, "Surrogate data for hypothesis testing of physical systems," Physics Reports, vol. 748, pp. 1 - 60, 2018. 